This is the peer reviewed version of the following article:

Otte M.A., Garcia-Martin A., Borrise X., Sepulveda B.. Metamirrors Based on Arrays of Silicon Nanowires with Height Gradients. Advanced Optical Materials, (2017). 5. 1600933: - . 10.1002/adom.201600933,

which has been published in final form at https://dx.doi.org/10.1002/adom.201600933. This article may be used for non-commercial purposes in accordance with Wiley Terms and Conditions for Use of Self-Archived Versions. 


\section{Metamirrors based on arrays of silicon nanowires with height gradients}

M. A. Otte, ${ }^{1}$ A. Garcia-Martin ${ }^{2}$, X. Borrise ${ }^{1}$, B. Sepulveda*1

${ }^{1}$ Catalan Institute of Nanoscience and Nanotechnology (ICN2), CSIC and The Barcelona Institute of Science and Technology, Campus UAB, Bellaterra, 08193 Barcelona, Spain

${ }^{2}$ IMM - Instituto de Microelectrónica de Madrid (CNM-CSIC), Isaac Newton 8, PTM, Tres

Cantos, E-28760 Madrid, Spain

*Corresponding Author: borja.sepulveda@icn2.cat

Keywords: Metamaterials, metamirrors, silicon nanowires

Metamaterials are artificial constructs that have attracted intense attention in the photonics field for finding new ways to control light propagation and to achieve complex optical effects.[1,2] To date, most metamaterials in the visible and near infrared regimes are based on arrays of nano-sized metallic, also known as nanoplasmonic, elements. Such plasmonic metasurfaces have not only achieved a remarkable versatility to control the optical properties in surfaces, but also shown interesting optical properties from the fundamental point of view such as hyperbolic ${ }^{[3,4]}$ or epsilon-near-zero ${ }^{[5]}$ effective dielectric constants. Plasmonic metamaterials have also enabled modifying light orbital angular momentum ${ }^{[6,7,8]}$ as well as the generation of the spin Hall effect, ${ }^{[9]}$ and have been already employed in actual applications such as holograms ${ }^{[10,11]}$ or wave retarders. ${ }^{[12]}$

The careful spatial control of size, orientation and shape of plasmonic nanostructures within the arrays has also been used to generate periodic phase gradients, which have given rise to the generalized Snell law to account for the 
anomalous reflection and refraction of light in these kind of metasurfaces. ${ }^{[13]}$ Such periodic phase gradients have been employed for the efficient coupling of light into surface modes, ${ }^{[14]}$ and for the development of metalenses. ${ }^{[15,16]}$

In the search of new metamaterials with low losses, high-index dielectric nanostructures exhibiting Mie resonances in the operation frequency range have emerged as a very appealing candidates. ${ }^{[17,18]}$ Silicon based metamaterials have been the base to create phase gradients by changing the geometry and distribution of the nanostructures on the surface. ${ }^{[19]}$ Silicon metamaterials have demonstrated applications as axicons, blazed gratings, achromatic metasurfaces and metalenses.[20]

Current plasmonic and dielectric metamaterials have in common that the desired optical response is achieved by a careful choice of the lateral size, shape, orientation and/or separation of the nanostructures on the surface. However, tailoring the vertical dimension of the nanostructures is generally cumbersome due to the technical complexity required to achieve high spatial resolution.

Herein we show that indeed a careful design of the nanostructure height is an efficient way to spatially tune the optical properties in high-index dielectric metamaterials. We demonstrate that arrays of silicon nanowires with carefully tailored height gradients generate phase gradients to develop metamirrors enabling light focusing in arbitrary shapes. In addition, the combination of height gradients and nanowires with anisotropic cross-section permits light focusing and strong polarization conversion in the focused light simultaneously.

We employ a recently developed fabrication method based on mechanically controlled metal assisted chemical etching of silicon ${ }^{[21]}$ to obtain ordered arrays of Si 
nanowires with height gradients. This fabrication method makes use of a very thin gold layer comprising an array of nanoholes with tunable mechanical properties as catalyst to etch the silicon substrate. During the etching process the catalytic metal mesh is plastically deformed, thereby generating the mechanical stress that spatially modulates the etching rate and gives rise to the array of nanowires with height gradients. The height gradients can be tuned by varying the stiffness of the catalytic metal mesh, i.e. by modifying the size and shape of the nanoholes array, the edge-toedge separation distance between nanoholes and the metal thickness. All the metamirrors shown in this work are produced using $20 \mathrm{~nm}$ thick gold films and the same etching conditions of Ref. [21].

The Si nanowire metamirror concept is shown in Figure 1a, which displays a circular array of cylindrical nanowires with height gradients in the radial direction, i.e. the height of the nanowires increases towards the center of the array. In the actually fabricated example of Figure 1b, the diameter of the array is $10 \mu \mathrm{m}$, the pitch is 300 $\mathrm{nm}$, and the nanowires height increases from zero at the border of the array, up to 500 $\mathrm{nm}$ at its center. In all the arrays in this work the $20 \mathrm{~nm}$ Au film used for etching is kept at the bottom of the array to increase the reflectivity and efficiency of the metamirror. In these arrays, the radial height variation is converted into a radial phase variation in the light reflected by the array. As Figure 1d shows, such radial phase gradient enables a tight focusing of the reflected light within a spot with a focal length of $12 \mu \mathrm{m}$. Since the nanowires have circular cross-sections, the focusing effect in Figure 1 is polarization insensitive. 

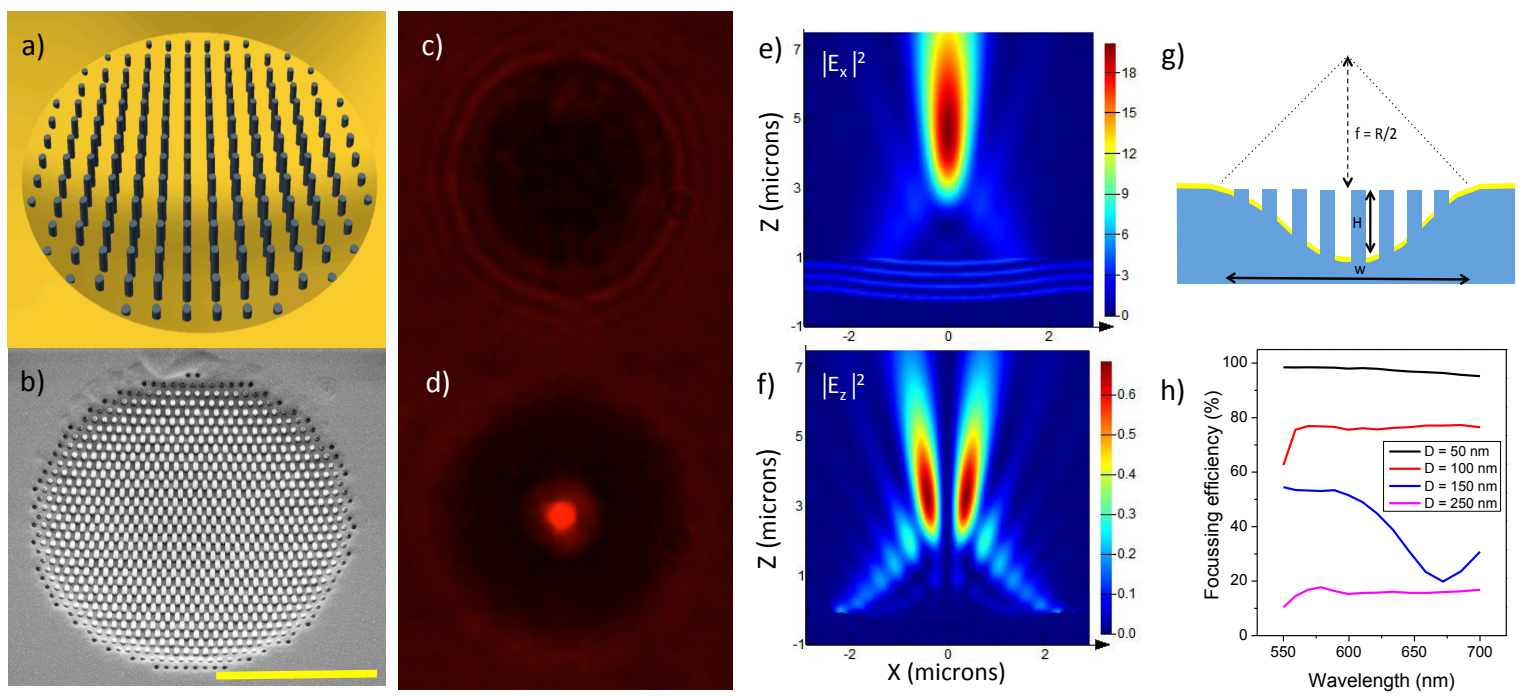

Figure 1 a) Schematic of a circular array of nanowires with height gradient in the radial direction. b) SEM image of a circular array of Si nanowires with height gradients in the radial direction. The array has a diameter of $10 \mu \mathrm{m}$, the diameter of the nanowires is $130 \mathrm{~nm}$ and the pitch is $300 \mathrm{~nm}$ (the scale bar is $5 \mu \mathrm{m}$ ). c) Optical image of the same array at the surface of the sample. d) Optical image of the focused reflected light by the array of nanowires with phase gradient in the radial direction. The focal length is $12 \mu \mathrm{m}$. e) and f) Spatial distribution of the squared absolute values of the $E_{x}$ and $E_{z}$ components of the reflected light by the array of $S i$ nanowires with radial height gradient, respectively. The diameter of the array is $5 \mu \mathrm{m}$, the pitch is $300 \mathrm{~nm}$, and the nanowires diameter is $50 \mathrm{~nm}$. The maximum height of the nanowires at the center of the array is $300 \mathrm{~nm}$. g) Schematic of the Si nanowire array and the equivalence with a spherical mirror of focal length $f$. h) Focusing efficiency of the metalenses compared to that of a silicon spherical mirror with $20 \mathrm{~nm}$ gold film, for nanowire diameters $D$ ranging from $50 \mathrm{~nm}$ to $250 \mathrm{~nm}$. 
Similar focusing behavior can be theoretically observed in FDTD calculations of circular arrays of cylindrical nanowires with equivalent radial height gradients. Due to computational limitations given by the large size of the computational volume, the size of the arrays in the calculations is restricted to $5 \mu \mathrm{m}$ in diameter. Figures $1 \mathrm{e}$ and $1 \mathrm{f}$ display the squared absolute value of the $E_{x}$ and $E_{z}$ components of the reflected light assuming an incident plane wave propagating in the $z$-direction and polarized in the $x$ direction. The focused spot can be seen in the amplitude of the $E_{X}$ component of the reflected electromagnetic field, whereas $E_{z}$ shows two lobes with zero intensity at the axis of the mirror. Since the nanowires have circular symmetry, the reflected light keeps the same polarization plane and the $E_{y}$ component is zero.

The diameter of the nanowires plays an important role in the focusing efficiency. The focusing effect is broadband for nanowires with diameters equal or less than 50 $\mathrm{nm}$, since the absorption by these nanowires is negligible in the visible. In these conditions the metamirror focal length barely changes with the light wavelength, and the focal length $f$ is similar to that of a spherical mirror, which is given by:

$$
f=\frac{R}{2}=\frac{H}{4}+\frac{W^{2}}{16 H}
$$

where $R$ is the radius of the spherical mirror, $H$ is the height of the nanowires at the center of the array and $W$ is the diameter of the array (Figure $1 \mathrm{~g}$ ). The focal length in the calculations of Figure 1e is $5 \mu \mathrm{m}$, and the focusing efficiency in the visible are practically equal those of the spherical mirror (Figure $1 \mathrm{~h}$ ). When the diameter of the nanowires is enlarged, the absorption efficiency increases, first at short wavelengths, and then in the whole visible spectrum. In arrays of nanowires with diameter larger 
than $200 \mathrm{~nm}$, the focusing efficiency is largely reduced and most of the visible light is absorbed by the array, as can be seen in Figure $1 \mathrm{~h}$.

A remarkable feature of the employed nanofabrication technique is its capacity to generate arrays with height gradients in arbitrary directions by changing the shape of the nanoholes array in the catalytic gold mesh.[21] Such capability is illustrated in Figure 2, where we show ring-shaped arrays of nanowires that focus the light in a ring with focal length of $10 \mu \mathrm{m}$. As shown above circular arrays of nanowires present broadband focusing for $50 \mathrm{~nm}$ nanowires (Figure 2b). In contrast, the focusing efficiency in the blue decreases when the nanowires diameter is $110 \mathrm{~nm}$ due to the enhanced absorption in this region (Figure 2d). In both cases the focal length is $10 \mu \mathrm{m}$, thereby fitting the expected focal length of a cylindrical mirror. In addition, Figures $2 \mathrm{e}$ and 2 f show how light can be focused using arrays with virtually any shape. Interestingly, these metamirrors with a lateral size of the array of nanowires of $2.5 \mu \mathrm{m}$ and height variations from zero to $120 \mathrm{~nm}$ are able to achieve a focal length as low as $3 \mu \mathrm{m}$, thereby demonstrating the capacity to tune the shape and the focal length with the geometry of the metamirror. 

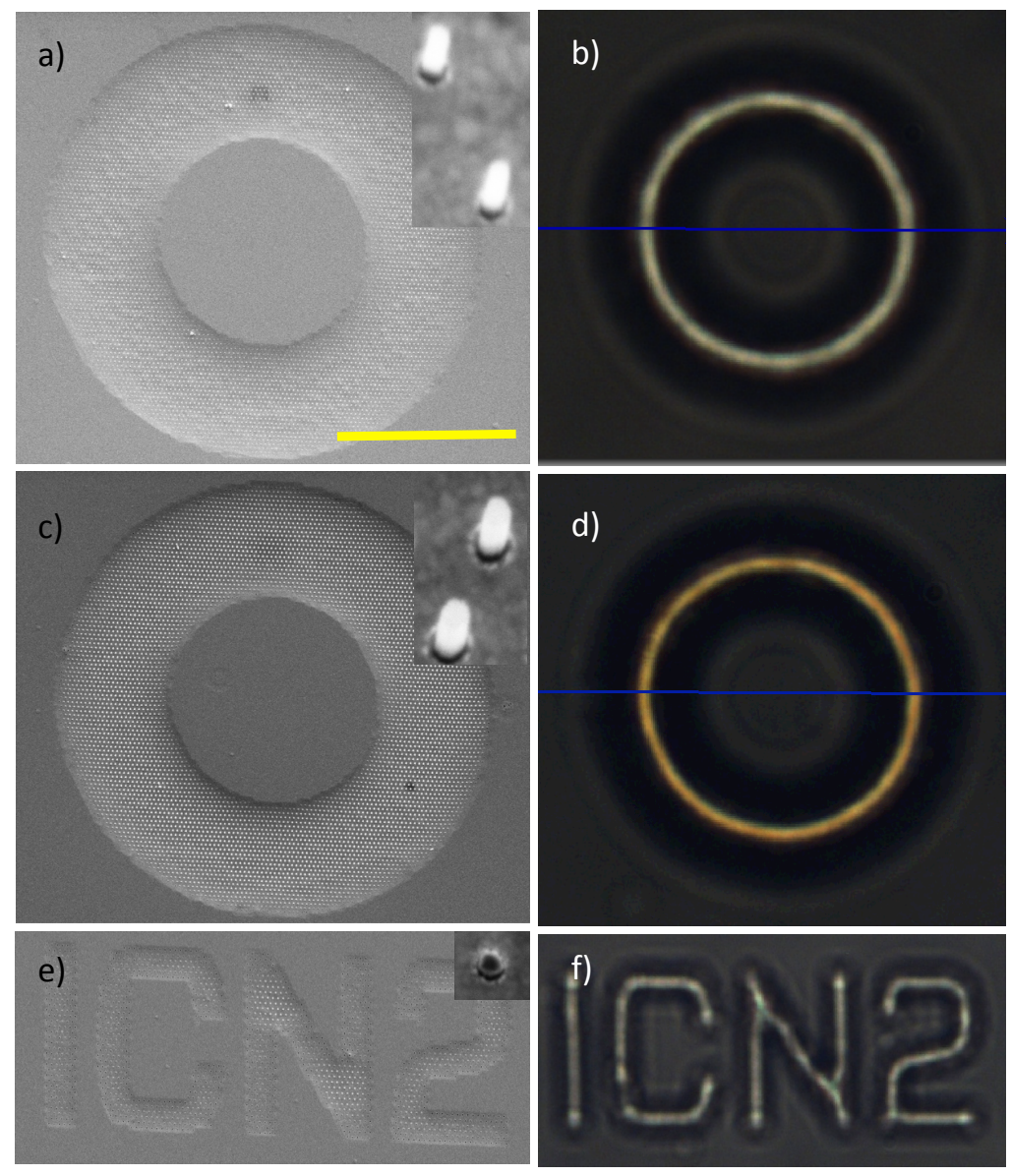

Figure 2. a) SEM images of a ring-shape metamirror (scale bar $10 \mu \mathrm{m}$ ). The internal and external diameters of the array are $7.5 \mu \mathrm{m}$ and $15 \mu \mathrm{m}$, respectively, and the diameter of the nanowires is $50 \mathrm{~nm}$ and their maximum height is $320 \mathrm{~nm}$. b) Optical image of the focused light by the ring with focal length of $10 \mu \mathrm{m}$. c) and d) SEM and optical images of a similar ring-shaped metamirror but for a nanowire diameter of $110 \mathrm{~nm}$ and a maximum height of $340 \mathrm{~nm} . \mathrm{e}$ ) and f) SEM and optical images of ICN2-shaped metamirror showing a focal length of $3 \mu \mathrm{m}$. The lateral size of the arrays is $2.5 \mu \mathrm{m}$, the nanowires diameter is $150 \mathrm{~nm}$ and their maximum height is $120 \mathrm{~nm}$. The optical images are taken using collimated white light from a halogen lamp. In all the cases the incident light is a collimated broadband light beam from a halogen lamp. 
All the metamirrors described so far are geometrically isotropic, i.e. the resulting focused light preserves the polarization of the impinging wave. However, the ability to combine nanowires with arbitrary cross sections and height gradients within the arrays opens up the opportunity to exert some degree of control on the polarization of the focused light. This is shown in the FDTD calculations of Figure 3 that show intense polarization conversion when the long axis of the nanowires is oriented at 45 degrees with respect to the polarization of incident light. By comparing the intensity of the $E_{x}$ and $E_{y}$ components at the focus to that of $E_{x}$ when the long axes of the nanowires are parallel to the polarization of incident light, a $30 \%$ conversion efficiency is predicted at $600 \mathrm{~nm}$ light wavelength, and close to $20 \%$ at $660 \mathrm{~nm}$.

These polarization conversion effects can be experimentally observed, as depicted in Figures 3b-3d for circular arrays of nanowires with elongated cross section and radial height gradient. When the short or long axes of the nanowires are parallel to the polarization of the incident light, the focused reflected light keeps the same polarization. Consequently, focused spots with slightly different intensity are observed when the analyzer is parallel to the incident light polarization, but the reflected light is totally blocked when the analyzer is oriented perpendicularly. In contrast, when the nanowires are aligned at 45 degrees with respect the incident polarization the focused spot is clearly seen for both orientations of the analyzer, thereby demonstrating the polarization conversion effect. Comparison of the focalized intensity in both analyzer orientations demonstrates that 30\% conversion efficiency at $660 \mathrm{~nm}$ is achieved. 

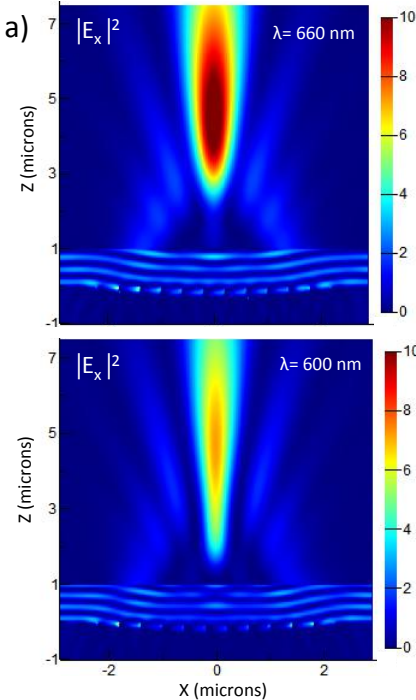
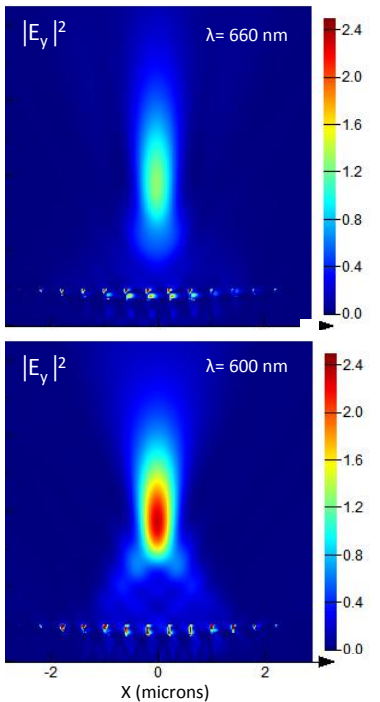

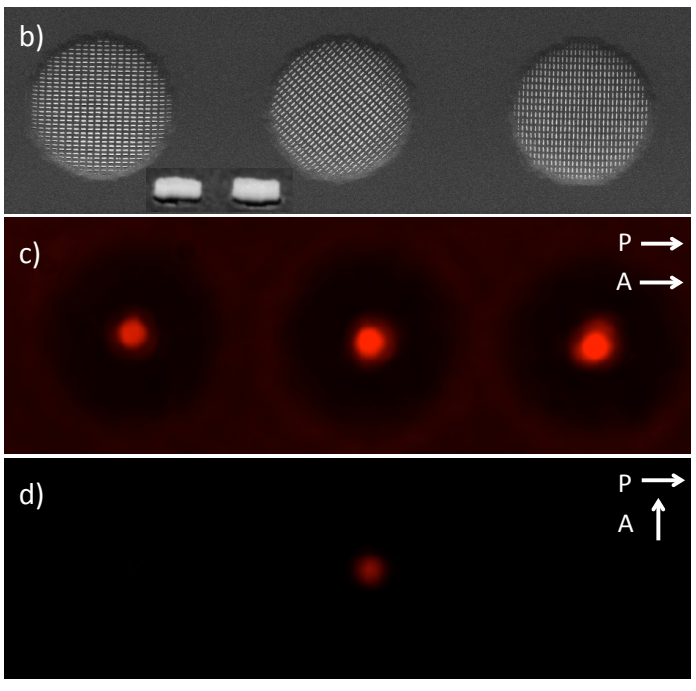

Figure 3. a) FDTD calculations anisotropic metamirror formed by an array of nanowires with ellipsoidal cross-section and radial height gradients showing the squared absolute values of $E_{x}$ and $E_{y}$ when the incident light is $660 \mathrm{~nm}$ and $600 \mathrm{~nm}$. The short and long axis of the nanowires are $250 \mathrm{~nm}$ and $100 \mathrm{~nm}$ respectively and are oriented at 45 degrees with respect to light polarization, the array diameter is $5 \mu \mathrm{m}$ and the nanowires height at the center of the array is $300 \mathrm{~nm}$. The pitch parallel and perpendicular to the long axis of the nanowires is 400 $\mathrm{nm}$ and $300 \mathrm{~nm}$, respectively. b) SEM image of circular metamirrors with $10 \mu \mathrm{m}$ diameter and elongated nanowires with the long axis oriented at 0 (left), 45 (center) and 90 degrees (right) with respect to the polarization of the incident light. The long and short axes of the nanowires are $250 \mathrm{~nm}$ and $100 \mathrm{~nm}$, respectively. The pitch in the array is $300 \mathrm{~nm}$ parallel to the short axis and $400 \mathrm{~nm}$ parallel to the long axis. c) Optical image of the focused light by the arrays when the incident light polarizer $P$ and analyzer $A$ of the reflected light are aligned. The incident light is a collimated $660 \mathrm{~nm}$ light beam from an LED lamp. d) Optical image of the focused reflected when the analyzer $A$ is rotated 90 degrees with respect to the incident light polarizer $P$. 
In conclusion, we have introduced arrays of silicon nanowires with height gradients as an interesting way to create optical metamaterials capable of light texturing and to develop a new generation of metamirrors able to focus light in complex patterns and, simultaneously, spatially control light polarization. Mechanical transfer of the arrays of $\mathrm{Si}$ nanowires to low refractive index substrates could also enable developing metalenses for transmitted light in the visible and infrared. Such metalenses would fully exploit the tunable Mie multipolar resonances and scattering patterns of silicon nanowires, ${ }^{[22,23,24]}$ which are strongly damped when they are on a silicon wafer, to achieve stronger lensing effects. Such silicon nanowire metalenses could find remarkable applications. On one hand, the possibility to fabricate arrays of metalenses with tailored geometries could be employed for efficient light coupling in fiber bundles. On the other hand, the demonstrated ability to focus the light in arbitrary shapes could be applied for light coupling in integrated optical circuits and optical fibers, and for the controlled efficient excitation of high order guided modes. Finally, polymer negative replicas of the Si nanowire metamirrors by nanoimprint techniques also offer a low cost alternative for developing new arrays of metalenses with very short focal lengths.

\section{MATERIALS AND METHODS}

\section{Fabrication of the silicon nanowire metamirrors}

First, a titanium/gold bilayer $(0.5 \mathrm{~nm} / 20 \mathrm{~nm})$ is evaporated on top of piranhacleaned silicon wafers (100) via electron beam deposition, on which a polymethyl 
methacrylate (PMMA $950 \mathrm{~K}, 6 \%$ in Anisole) resist layer is spin coated (4000 rpm: thickness $\approx 300 \mathrm{~nm}$ ). Then, electron beam lithography (EBL) is used to pattern arrays of nanoholes of different geometries into the resist. After EBL exposure the resist is developed during $40 \mathrm{~s}$ in a mixture of methyl isobutyl ketone (MIBK) and isopropyl alcohol (IPA) at a ratio of 1:3. Immersing the chip in IPA stops the process, and after that it is blown dry with $\mathrm{N}_{2}$ flow.

The patterned PMMA layer is used as a mask for the removal of the exposed gold inside the holes by directional argon sputtering treatment (ICP RIE, power 1000 $\mathrm{W}$, chuck power $25 \mathrm{~W}$, pressure $1 \mathrm{~Pa}$, flow $20 \mathrm{sccm}$, time $50 \mathrm{~s}$ ). During this process the approximately $300 \mathrm{~nm}$ thick PMMA layer is strongly damaged, but it is still sufficiently thick to protect the gold layer underneath. Subsequently, the remaining PMMA is removed via an oxygen reactive ion etching (ICP RIE power $500 \mathrm{~W}$, pressure $1 \mathrm{~Pa}$, flow $20 \mathrm{sccm}$, time $240 \mathrm{~s}$ ), leaving a clean, patterned $20 \mathrm{~nm}$ thick gold layer.

The silicon nanopillars are finally created via metal-assisted chemical etching (MACE), by immersing the sample into a mixture of $\mathrm{H}_{2} \mathrm{O}(89,5 \%), \mathrm{H}_{2} \mathrm{O}_{2}(0.5 \%)$ and $\mathrm{HF}$ (10\%) for 2 min. To stop the MACE, the samples are immersed in water. Finally, the samples are transferred to IPA to minimize capillary effects upon drying with $\mathrm{N}_{2}$ flow.

\section{Optical characterization of the metamirrors}

A custom made optical set up has been used to optically characterize the metamirrors. The sample is mounted on a motorized stage that enables a controlled variation of the $\mathrm{Z}$ distance with the respect to the microscope objective. Additional manual stages are used to move the sample in the XY directions. The light from either

a fiber coupled LED (Thorlabs) with central emission wavelength at $660 \mathrm{~nm}$ or a 
halogen lamp (Ocean Optics) is collimated by an achromatic lens, passes through a lineal polarizer and a 50/50 beam splitter, and, using a second achromatic lens, is finally focused on the back focal plane of an ultra long working distance 100X Nikon objective, to achieve collimated light at the output. The reflected light by the sample travels through a tube lens and a second polarizer (analyzer) and is then captured by a Nikon DFSI1 CCD camera. The motorized Z stage enables focusing the surface of the sample on the CCD camera, as well as imaging the focused spots and measuring the focal length of the metamirrors.

\section{FDTD calculations}

The FDTD calculations have been performed using commercial Lumerical software. The mesh resolution in the region of the nanowires and the metal film is 5 $\mathrm{nm}$, and we have employed a total field scattered field source incident on the metamirror and PML boundary conditions. To avoid undesired instabilities caused by the metal film at short wavelengths, the calculations of the metamirrors are restricted to the $550 \mathrm{~nm}$ to $700 \mathrm{~nm}$ spectral range.

\section{ACKNOWLEDGEMENTS}

Authors acknowledge the financial support of Spanish MINECO projects MAT2013-48628-R, FIS2013-49280-EXP and MAT2011-12645-E, and Comunidad the Madrid project S2013/MIT-2740. 


\section{REFERENCES}

[1] N. Yu, F. Capasso, Nat. Mater. 2014, 13, 139.

[2] A. V. Kildishev, A. Boltasseva, V. M. Shalaev, Science 2013, 339, 1289.

[3] A. Poddubny, I. Iorsh, P. Belov, Y. Kivshar, Nat. Photon. 2013, 7, 958.

[4] A. A. High, R. C. Devlin, A. Dibos, M. Polking, D. S. Wild, J. Perczel, N. P. de Leon, M. D. Lukin, H. Park, Nature 2015, 522, 192.

[5] R. Maas, J. Parsons, N. Engheta, A. Polman, Nat. Photon. 2013, 7, 907.

[6] N. Shitrit, I. Yulevich, E. Maguid, D. Ozeri, D. Veksler, V. Kleiner, E. Hasman, Science 2013, $340,724$.

[7] G. Li, M. Kang, S. Chen, S. Zhang, E. Y. Pun, K. W. Cheah, J. Li, Nano Lett. 2013, 13, 4148.

[8] E. Karimi, S. A. Schulz, I. De Leon, H. Qassim, J. Upham, R. W. Boyd, Light Sci. Appl. 2014, 3, e167.

[9] X. Yin, Z. Ye, J. Rho, Y. Wang, X. Zhang, Science 2013, 339, 1405.

[10] L. Huang, X. Chen, H. Mühlenbernd, H. Zhang, S. Chen, B. Bai, Q. Tan, G. Jin, K. Cheah, C. Qiu, J. Li, T. Zentgraf, S. Zhang, Nat. Commun. 2013, 4, 2808.

[11] G. Zheng, H. Mühlenbernd, M. Kenney, G. Li, T. Zentgraf, S. Zhang, Nat. Nanotech. 2015, $10,308$.

[12] N. Yu, F. Aieta, P. Genevet, M. A. Kats, Z. Gaburro, and F. Capasso, Nano Lett. 2012, $12,6328$.

[13] N. Yu, P. Genevet, M. A. Kats, F. Aieta, J. Tetienne, F. Capasso, Z. Gaburro, Science 2011, $334,333$.

[14] S. Sun, Q. He, S. Xiao, Q. Xu, X. Li, L. Zhou, Nat. Mater. 2012, 11, 426.

[15] X. Ni, S. Ishii, A. V. Kildishev and V. M. Shalaev, Light: Sci. Appl. 2013, 2, e72.

[16] S. Ishii, V. M. Shalaev, A. V. Kildishev, Nano Lett. 2013, 13, 159.

[17] A. García-Etxarri, R. Gómez-Medina, L. S. Froufe-Pérez, C. López, L. Chantada, F. Scheffold, J.

Aizpurua, M. Nieto-Vesperinas, J. J. Sáenz, Opt. Express 2011, 19, 4815.

[18] N. de Sousa, L. S. Froufe-Pérez, J. J. Sáenz, A. García-Martín, Sci. Rep. 6, 2016, 30803.

[19] D. Lin, P. Fan, E. Hasman, M. L. Brongersma, Science 2014, 345, 6194..

[20] F. Aieta, M. A. Kats, P. Genevet, F. Capasso, Science 2015, 347, 1342.

[21] M. A. Otte, V. Solis-Tinoco, P. Prieto, X. Borrisé, L. M. Lechuga, M. U. González, B. Sepulveda, Small 2015, 11, 4201.

[22] M. Nieto-Vesperinas, R. Gomez-Medina, and J. J. Saenz, J. Opt. Soc. Am. A 2011, $28,54$.

[23] J. M. Geffrin, B. García-Cámara, R. Gómez-Medina, P. Albella, L. S. Froufe-Pérez, C. Eyraud, A. 
Litman, R. Vaillon, F. González, M. Nieto-Vesperinas, J. J. Sáenz, F. Moreno, Nat. Commun. 2012, 3, 1171.

[24] B. García-Cámara, R. Gómez-Medina, J. J. Sáenz, and B. Sepúlveda, Opt. Express 2013, 21, 23007. 